Published in final edited form as:

Community Dent Oral Epidemiol. 2007 August ; 35(Suppl 1): 41-49. doi:10.1111/j. 1600-0528.2007.00404.x.

\title{
Concurrent validity of the COHIP
}

\author{
Neil Dunlow ${ }^{1}$, Ceib Phillips ${ }^{2}$, and Hillary L. Broder ${ }^{3}$ \\ ${ }^{1}$ US Air Force, Dental Corp, Omaha, NE, USA \\ ${ }^{2}$ Department of Orthodontics, University of North Carolina Chapel Hill, NC, USA \\ ${ }^{3}$ Hunterdon Endowed Professor, Department of Community Health, University of Medicine \& \\ Dentistry of NJ, Newark, NJ, USA
}

\begin{abstract}
Objectives-This study examined the relationship between children's perception of their OHRQOL and their perceptions of their dentofacial image, social anxiety, and self-concept as an assessment of the concurrent validity for the Child Oral Health Impact Profile (COHIP).
\end{abstract}

\begin{abstract}
Methods-A nonrandom, consecutive sample of children, ages nine to 14 years, was recruited for this observational validation study. Participants had been accepted for treatment in the University of North Carolina Graduate Orthodontic clinic. Data were collected after gathering initial orthodontic records and prior to delivery of any fixed or removable orthodontic appliances. Participants completed the $C O H I P$ and standardized dimension-specific questionnaires with known psychometric properties designed to assess self-concept, social anxiety, and perception of facial image. Child assent with caregiver consent was obtained prior to data collection. Pearson's correlations between each of the domains of the COHIP and the Dento-facial Image, the Social Anxiety Scale, and the self-concept domains of the Multidimensional Self Concept Scale (MSCS) were calculated. Criteria for support of concurrent validity was established based on directionality of expected relationships and strength of the observed correlation coefficient. Each correlation was assessed as meeting or not meeting the criteria. A one-tailed one sample $Z$-test was used to test the null hypothesis that $58 \%$ of the calculated correlations would meet the criteria (expected a priori) with an alterative that less than $58 \%$ would meet the criteria.
\end{abstract}

Results-The average age of the 52 subjects enrolled was $11.8 ; 40 \%$ were male; and $85 \%$ were Caucasian. The hypothesis that $58 \%$ of the calculated correlations defined a priori as expected relationships would meet the criteria was supported by the data $(P=0.63)$. The perception of dentofacial appearance was positively correlated (range $=0.39$ to 0.45 ; with all of the $C O H I P$ domains except for the School domain. Overall, the COHIP domains, particularly Self-Image and Social Emotional subscales, were positively correlated (0.32-0.52) with the MSCS self-concept domain scores, except Family Self-Concept. The COHIP domains, particularly Functional Wellbeing and Social Emotional subscales, were negatively correlated ( -0.76 to -0.33$)$ with the three Social Anxiety subscales that include both fear of negative evaluation and social avoidance.

Conclusions-The findings in this study lend support to the validity of the COHIP since $77 \%$ of the expected relationships between the domains of the COHIP and the domains of general, standardized dimension-specific instruments were observed. The decision to use conditionspecific, dimension-specific, or general quality of life (QOL) measures is dependent on the purpose of the study. For investigations in children on the effect of dental treatments or in 
epidemiologic studies of an oral health outcome, the use of condition-specific QOL measures like the COHIP have the advantages of increased patient responsiveness since the assessment is focused on a specific condition, oral health, and increased sensitivity to treatment effects.

\section{Keywords}

child oral health impact profile; facial image; Oral health-related quality of life; self-concept; social anxiety

In 2000, the Surgeon General's report and conference entitled 'The Face of the Child: Children and Oral Health' highlighted the importance of children's oral health to their overall health and well-being and the profound impact that oral health can have on the quality of children's lives (1). Oral health-related quality of life has been investigated in adults and adolescents. However, it has not been extensively investigated in children (2), largely because of the lack of a developmentally appropriate, validated instrument $(3,4)$. The Child Oral Health Impact Profile (COHIP) was developed specifically to address the methodological issues outlined for this population: the instrument assesses the four theoretically relevant dimensions or domains when considering children's OHRQOL and can be used in self-report format for children aged 8-16 $(5,6)$. See manuscript by Broder et al. in this issue for more detail.

The principal aim of this investigation was to assess the concurrent validity of the COHIP domains in a population other than the one used in its development. Concurrent validity testing is, by definition, conducted in the absence of a universally accepted standard for comparison. Validity of a new instrument like the COHIP is ascertained by examining the correlations between the COHIP domains and the scores from other instruments, administered concurrently, that are expected to be related to these domains because the comparison instruments assess similar underlying constructs (7-9). This validation approach is recommended and has been used in widely diverse health areas: for example, eye allergy (10); disordered eating attitudes in children (11); affective and anxiety problems in youth (12); Crohn's Disease (13); asthma (14); and COPD (15). The intent of this study was to examine the relationships between children's perceived impact of their oral health on different aspects of their lives (Oral Health; Functional Well-being; Social-Emotional Well Being; School-Environment; Self-Image) and their perception of their dentofacial attractiveness, social anxiety, and self-concept.

\section{Procedures and instruments}

The project was approved by the Institutional Review Board at the School of Dentistry at the University of North Carolina. A nonrandom, consecutive sample of children, male and female, ages nine to 14 years, was recruited who had been accepted for treatment in the graduate orthodontic clinic at the University of North Carolina's School of Dentistry. Data were collected after gathering initial orthodontic records and prior to delivery of any fixed or removable orthodontic appliances.

Subjects were eligible if they were enrolled in the fourth through eighth grade in school; were able to read at a third grade level; and presented with a mild to moderate malocclusion not requiring orthognathic surgery. Subjects were excluded if they had been or were being prescribed medications to alter mood; had a systemic medical condition that might affect physical or emotional growth; had a congenital syndrome or previous orthognathic surgery procedure; or had had removable or fixed appliance(s) previously.

Following consent, the patient-based data were collected. Since the development of the COHIP indicated an impact of oral health on oral function, socioemotional well-being, 
school and peer functioning, and self-concept, instruments developed for use in the same age group that purport to assess more generally the child's self-concept, social anxiety, and perception of attractiveness were selected as comparison instruments. Uniform verbal instructions were given to each subject, and during completion of the questionnaires, the subject was seated in a quiet room or a quiet area apart from caregivers and other subjects. Assistance regarding comprehension of questions was available.

Overjet and overbite were measured as simple clinical indicators of the child's malocclusion and the child was asked to self-rate their own need for orthodontic treatment using the patient IOTN-AC. (16). These measures were obtained to characterize the severity of the malocclusions observed in the sample. The IOTN - AC was developed to provide a reliable method of ranking malocclusions based on certain occlusal characteristics. Subjects were asked to identify the image out of a set of 10 photographs, without the aid of a mirror, photograph, or model, that (s)he thought most closely represented their pre-treatment dental arrangement. Photographs were presented to subjects in random order although the photographs can be rank ordered from least dentally attractive (1) to most dentally attractive (10).

Subjects were paid $\$ 10$ for completing data collection. One-hour parking vouchers were given to caregivers to help defray the cost of parking during data collection.

\section{Instruments}

Child oral health impact profile $(5,6)$-The COHIP questionnaire was developed to measure OHRQOL as reported by children who are at least 8 years of age. The questionnaire consists of 34 items. Domain construction resulted in the following six subscales: Oral Health, Functional Well-Being, Social-Emotional Well-being, SchoolEnvironment, Self-Image, and Treatment Expectations. If more than two-thirds of the items on a subscale are missing the score is not computed. Scores are computed as the sum of the responses on that subscale.

Dentofacial image (subscale of the FI) (17)—Subjects rate on a 1 ('Have Strong Negative Feelings') to 5 ('Have Strong Positive Feelings') scale features related to the perioral region (nose, lips, mouth, teeth, chin, profile, and smile). The Dentofacial Image (DFI) score was computed as the average of the responses to the seven features. A higher score indicates that a subject has generally positive feelings about his or her facial image.

Multidimensional self concept scale (MSCS) (18)—The MSCS is based on a hierarchical model of self-concept that assumes the multiple dimensions are moderately intercorrelated and that each dimension contributes to the child's global self-concept. The $M S C S$ was designed for use with children aged 9-19 and has excellent reliability and validity. Cronbach's alpha exceeds .88 for all subscales and the total score.

This questionnaire contains various statements pertinent to six domains - the $S$ (social) Scale, the C (competence) Scale, the AFF (affect) Scale, the AC (academic) Scale, the F (family) Scale, and the P (physical) Scale. Children completing the MSCS indicate the degree to which they agree with each statement by selecting an answer of 'Strongly Agree,' 'Agree,' 'Disagree,' or 'Strongly Disagree.' Each domain consists of 25 items, each scored from 1 to 4 . Negatively worded items were reverse-scored prior to calculating the sum of the raw scores for each domain. The raw domain scores were then standardized using the standard score conversions available in the user manual on an IQ metric with a mean of 100 and a standard deviation of 15 . Higher scores indicate higher self-concept in that domain. 
Social anxiety scale (19)—The $S A S$ scales were developed to assess children's and adolescents' feelings of social anxiety in the context of their peer relations. Subjects in fourth through sixth grades completed the Children's - Revised version $(S A S C-R)$ and subjects in the seventh or eighth grade completed the Adolescent's version $(S A S-A)$. Each version consists of statements pertaining to feelings about social interactions. The two versions have similar or identical statements and the same three subscales apply. Cronbach's alpha for the three subscales exceed 0.69 in unselected school populations and 0.6 in clinical populations. $F N E$ (fear of negative evaluation from peers) consistently has the highest internal consistency of the three subscales $(>0.85)$.

Participants indicate how much they feel that each statement is true for them, and possible answers are 'Not At All,' 'Hardly Ever,' 'Sometimes,' 'Most of the Time,' and 'All the Time.' Three subscales are calculated: $F N E$, 'fear of negative evaluation from peers'; $S A D$ $N e w$, social avoidance and distress that is specific to new situations or unfamiliar peers; and $S A D$-General, 'social avoidance and distress that is experienced more generally when in the company of peers.' Higher scores indicate higher social anxiety. Twenty-one (40\%) of the subjects completed the $S A S C-R$ version.

\section{Predicted relationships}

The comparison instruments were selected on the basis of their excellent reliability and validity reported for the age range appropriate for usage of the COHIP and were believed to tap conceptually similar constructs as the COHIP. These instruments were developed as dimension-specific instruments intended to assess a particular construct (social anxiety, selfconcept, perception of facial image) from a general perspective rather than a conditionspecific perspective. Since these instruments have a limited application in dentistry, it is difficult to specify, a priori, expected levels of correlation. However, the following expected relationships were constructed based on a comparison of the comparison questionnaire's published intent and description and an examination of the similarity in wording or construct between scales of the comparison questionnaires and COHIP. In general, positive correlations were expected between the perception of Dentofacial Image, Physical Selfconcept, Social Self-concept, Affect Self-concept and all of the COHIP domains, except Treatment Expectancies (Table 1). The hypothesis was that the more positive the feelings a child had about his/her oral health and its impact on life activities then the more positive would be the child's perception of his/her dentofacial attractiveness; his/her physical self which is affected by the child's comparisons of his/her physical attributes to others as well as the reaction of others; his/her social self based on social interactions with family, classmates, and others; and previous and current behaviors as viewed by the child and reinforced by others (affect self-concept).

Negative correlations between $C O H I P$ domains and all three subscales of social anxiety were expected based on the likelihood that the more negative the feelings a child had about his/her OHRoL then the more anxiety relating to social interactions a child would report (Table 1).

No associations with the Treatment Expectations domain were expected since this domain seeks beliefs about future health resulting from treatment that are of a different nature from the psychosocial perceptions solicited by the comparison questionnaires. No associations between the COHIP domains and the Competence, Academic, and Family Self-concept domains were expected under the assumption that the instruction to base their response based on how their feelings about their teeth and mouth impacted their daily activities was too specifically focused to be related to these $M C S C$ domains. 


\section{Statistical analysis}

Simple bivariate correlations between each of the domains of the COHIP and the Dentofacial Image, the three Social Anxiety constructs, and the $M D S C$ self-concept domains were calculated. We hypothesized that relationships with the Social Anxiety constructs would be negative and that all other relationships would be positive. To be considered supportive of the hypothesized relationships (Table 1), the directionality of the relationship and an absolute value Pearson correlation coefficient of at least 0.3 was required. This level corresponds to a medium effect size and implies that approximately $10 \%$ of the variance in the dimension specific domain is attributable to the variance in the COHIP response (20). Satisfaction of this requirement was interpreted to mean that the relationship between the OHRQOL construct and the more general psychosocial construct supported the validity of the COHIP domain. The low criterion value $(r=$ absolute value of 0.30$)$ was set because the dimension-specific and condition-specific (COHIP) instruments, although assessing similar constructs, are not measuring precisely the same framework of perceptions. There is no general accepted criteria for the strength of the correlation as supportive evidence of concurrent validity $(21,22)$. This level of correlation as an indicator of support for validation has been used in other studies $(10,11,13,14)$. Higher correlations would have been expected if another OHRQoL instrument had been available for comparison. For each of the 60 correlation coefficients calculated, an indicator value was set to 1 if the criteria for support (directionality and correlation value) was met and 0 otherwise. The one sample $Z$ test was used to test the null hypothesis that $58 \%$ of the correlation coefficients met the criteria compared to the one-tailed alternative that less than 58\% met the criteria. Level of significance was set at 0.05 .

\section{Results}

During the approximately 12-month period of subject recruiting and data collection, 124 eligible patients presented for initial records in the graduate orthodontic clinic at the University of North Carolina's School of Dentistry. Thirty-eight potential subjects either were not contacted for participation or did not complete data collection prior to delivery of orthodontic appliances. Thirty-four patients or parents declined to participate.

Fifty-two patients, who had given assent with parental/legal guardian consent, completed data collection. All data were gathered in conjunction with scheduled orthodontic visits in order to maximize convenience for subjects and caregivers. The average age of the subjects was 11.8 years, with a range from 9 years, 4 months to 14 years, 6 months. Slightly more than $40 \%$ of the subjects were male, and approximately $85 \%$ were Caucasian.

On average, the subjects tended to exhibit mild positive overjets with very mild deep bites. Average overjet was $4.3 \mathrm{~mm}$. Sixty percent of the children were mild class II with an overjet between 3 and 7 and 14\% were severe Class II (overjet $>7 \mathrm{~mm}$ ). None of the children had a reverse overjet. Seventy percent had mild to moderate positive overbite (3-7 mm). Fiftyfour percent of the children perceived no or only a mild need (IOTN-AC of 0-3) for orthodontic treatment. Thirty-nine percent perceived a moderate need for treatment (IOTNAC of 4-7) and $10 \%$ thought that their malocclusion was severe. Parental education level indicated, in general, a middle class socioeconomic background for sample participants. Seventeen caregivers (33\%) had completed the equivalent of a high school education, and nine (17\%) had finished some college training. Fourteen caregivers (27\%) were college graduates, while 12 parent/guardians (23\%) reported some post-college education.

Descriptive statistics for each of the questionnaires is provided in Table 2. The average COHIP subscales for this sample are quite similar to those of the previously reported orthodontic sample (6). The children's perception of their dento-facial attractiveness, on 
average, was more negative than was expected given the relative severity of the malocclusions. The average DFI was similar to that of older adolescent/adult patients treatment planned for camouflage orthodontics or orthognathic surgery. Relative to established norms (18), the mean overall $M S C S$ self-concept score prior to treatment was average. The mean domain scores for all six areas of self-concept were also in the average range. The children in this sample expressed less social anxiety, on average, than published norms (19).

Fifty-eight percent of the relationships between COHIP domain scores and the standardized dimension-specific scores were expected a priori to have a specific directionality (positive or negative) and to meet or exceed the correlation criterion value. Of the correlation coefficients observed, $60 \%$ met both the directionality and correlation value criteria (Tables 3 and 4 ). The $P$-value associated with the one-tailed one sample $z$-test was 0.63 supporting the null hypothesis that $58 \%$ of the relationships met the criterion. Of the 35 specific relationships expected to meet criterion, $27(77 \%)$ did. The other nine relationships that met criterion were not anticipated: all were moderate relationships ( $r$ ranged from 0.33 to 0.54 ) between the COHIPdomains and the Competence and Academic domains of the MSCS (Table 3).

The Pearson correlation coefficients between the subjects' COHIP domain scores and their scores on the MSCS and Dentofacial Image are provided in Table 3. As hypothesized, the perception of the dentofacial appearance was positively correlated with all of the $C O H I P$ domains except for the impact of oral health on the School domain items. Thus, negative feelings regarding the attractiveness of the dentofacial area were associated with the child's perception that his/her oral health negatively affected all areas of life except for performance in school.

All of the self-concept domain scores, except for Family Self-concept, were positively correlated with the majority of the COHIP subscales. The positive sign associated with these correlations indicates that subjects with more favorable perceptions of their OHRQOL also had higher self-concept in all of the MSCS domains except that regarding family relationships.

The FNE, SAD-New, and SAD-general subscales of the Social Anxiety Scale were negatively correlated with the COHIP domains except Treatment Expectations (Table 4). Higher levels of social anxiety corresponded with less favorable perceptions regarding OHRQOL (Table 4). Interestingly, the construct captured by the School-Environment Subscale of the COHIP was quite strongly associated with fear of negative evaluation and concern about social interactions in new situations or with unfamiliar peers but not with perception of self as indicated by DFI and MCSC domains.

\section{Discussion}

The principal aim of this study was to assess the concurrent validity of the COHIP in a population other than the one used in its development. The COHIP is a scale that was developed to be used across oral health conditions; therefore, it should be sensitive to the impact of malocclusion on OHRQOL. The findings support the validity of the COHIP. Further investigation will be required, given the sampling approach and ethnic, socioeconomic and mild malocclusion characteristics of the sample, to determine if groups of children differentiated by the type or severity of the malocclusion respond differently or if the COHIP is sensitive to changes following orthodontic or orthognathic surgery in children.

Evaluation of the concurrent validity of a new instrument is based on the support of theoretical relationships between the new instrument (COHIP) and other general, dimension- 
specific instruments that assess similar constructs. For example, since no unanimously accepted benchmark exists for evaluating the QOL effects of facial skeletal abnormalities, Cunningham and her co-investigators compared scores on the Orthognathic Quality of Life Questionnaire (OQLQ) $(9,23)$, a condition-specific QOL designed for use with patients who have severe disharmonies of the oral-facial complex and who are considering orthognathic surgery as a treatment option, to scores from a general HRQOL instrument, the $S F-36$ (24), and a 100-millimeter visual analog scale assessing perceptions about overall appearance and function. The actual (calculated) strength and direction of correlations between the subscales of the $O Q L Q$ and $S F-36$ and perceptions of appearance were compared to the hypothetical relationships in order to confirm the validity of the $O Q L Q(9)$.

The present investigation used a similar approach to validate the $C O H I P$ in a generally healthy population of subjects and in the absence of a 'gold standard' for comparison. Some of the expected relationships regarding the presence or absence of correlations and directionality of the correlation were based upon the types of questions contained within various subscales of the $C O H I P$ and the standardized, domain-specific questionnaires used in the current investigation and others on the underlying concept as indicated by the subscale designation. In concurrent validity testing, the emphasis is on the consistency of findings and the relative strength of the associations observed rather than on the $P$-value associated with each correlation coefficient as an inferential assessment $(21,22)$. For this reason, we chose to test a single hypothesis using the calculated correlation values as random variables in a global test of the percentage of correlations that met criteria for support of the concurrent validity of the COHIP. In this study, 77\% (27 of 35) of the expected relationships and nine of the $25(36 \%)$ relationships not anticipated a priori met the criteria of directionality and strength of the correlation for a total of $60 \%$ of the associations. The hypothesis that, as a set, $58 \%$ of the total number of relationships would meet criteria was supported. The School-Environment domain of the COHIP did not correlate as expected with the perception of dento-facial attractiveness or physical or social self-concept. It is likely that the construct captured by the School-Environment Well-being domain is unrelated to the child's perception of self but related to the perception of how others perceive him/her in that environment.

The social subscale of the MSCS contains statements that relate to children's social and peer interactions, such as 'I am too shy,' 'I often feel like I am left out of things,' and 'I have a lot of friends.' The affect subscale includes statements that pertain to children's overall mood. Examples include 'I enjoy life,' 'I feel like a failure,' and 'I feel loved.' Intuitively, more positive scores on the social and affect subscales would be expected to be associated with higher scores on the Social Emotional Well Being and Self-Image domains of the COHIP. These expectations were generally confirmed. The association between positive affect scores and more favorable assessments of OHRQOL has been reported previously (1, $25,26)$.

The competence subscale of the MSCS was designed to assess children's perceptions of their capabilities and proficiencies, and it contains statements such as 'I am very self confident,' 'I give people good reason to trust me,' and 'I can do things most things pretty well.' Based on the nature of these statements, higher estimations of competence might be expected to be associated with more favorable assessments of social and emotional wellbeing and self-image related to the oral condition. Relationships were not set a priori between the Competence Self-Concept and COHIP domains under the assumption that the instruction to base their response based on how their feelings about their teeth and mouth impacted their daily activities was too specifically focused. Perhaps positive feelings about oral health translate to a greater sense of confidence and competence overall. 
The physical scale of the MSCS includes statements like 'I am attractive,' and 'I feel good about how I look.' These statements are not directly related to the teeth or mouth, but given the importance of the face in the attractiveness literature, positive correlations were expected and observed. Positive feelings about the appearance of the dentofacial region would intuitively be expected to be associated with more favorable ratings of OHRQOL as it relates to social and emotional well-being, interactions in school, and self-image on the COHIP since the instructions for the COHIP ask about the impact of 'the teeth, mouth, or face.' The associations between $C O H I P$ and Dentofacial Image were observed as expected.

Scores on all three components of the Social Anxiety Scale (FNE, SAD-new, and SADgeneral) were expected to be associated with perceptions of the impact of the oral condition on social-emotional well-being, interactions in school, and self-image on the COHIP. Intuitively, less favorable perceptions about OHRQOL would be anticipated to lead to greater social anxiety. These relationships were observed as expected. Indeed, the strongest correlations between the standard, dimension-specific instruments and the COHIP were observed between social anxiety, expressed as a fear of how peers 'judge' you (FNE) or as the distress experienced during new social situations or when meeting new peers (SADSnew), and the impact of the oral condition on social and emotional well-being, functional well-being, and interactions in school. Negative feelings by a child about their oral condition may produce feelings of anxiety and shame that may inhibit an individual from interacting with peers in a positive way $(27,28)$. Conversely, problems with peers may lead to a child's heightened concerns about his/her oral condition.

\section{Conclusion}

In conclusion, the results suggest that the COHIP is a valid measure and is appropriate for use as a condition-specific assessment of the impact of oral health on the daily lives of children. Scores on the COHIP and the perception of the attractiveness of the dentofacial region and the self-concept, and social anxiety measures were generally correlated, as expected. Further concurrent validity studies in children with other oral health needs are warranted given the rather homogeneous sample in terms of oral problem and demographic characteristics included in this study. The decision to use condition- specific, dimensionspecific, or general QOL measures is dependent on the purpose of the study. For investigations in children on the effect of dental treatments or in epidemiologic studies focused on oral health impact, the use of condition-specific QOL measures like the COHIP have the advantages of increased sensitivity and relevance to the participants.

\section{Acknowledgments}

This study was supported in part by the National Institute of Dental and Craniofacial Research R01 DE 005215 and R01 DE13732.

\section{References}

1. National Institute of Dental and Craniofacial Research. Surgeon General's Conference on children and Oral Health. Washington, DC: Office of the Surgeon General, US Department of Health and Human Services; 2000. p. 12-13.

2. Weintraub J. Uses of oral health related quality of life measures in public health. Community Dent Health. 1997; 15:8-12. [PubMed: 9791608]

3. Jokovic A, Locker D, Stephens M, Kenny D, Tompson B, Guyatt G. Validity and reliability of a questionnaire for measuring child oral-health-related quality of life. J Dent Res. 2002; 81:459-63. [PubMed: 12161456]

4. Edwards TC, Huebner CE, Connell FA, Patrick DL. Adolescent quality of life, part I: conceptual and measurement model. J Adolescence. 2002; 25:275-86. 
5. Broder HL, Wilson M, Reisine S, Phillips C, Janal M. Reliability and validity of the child oral health impact profile (COHIP). J Dental Res. 2005; 84:2652. (Spec Iss A).

6. Broder HL, Wilson-Genderson M. Reliability and validity of the Child Oral Health Impact Profile (COHIP). Community Dent Oral Epidemiol. 2007; 35(Suppl 1):20-31. [PubMed: 17615047]

7. Guyatt, GH.; Jaeschke, R.; Feeny, DH.; Patrick, DL. Chapter 5: Measurements in clinical trials: choosing the right approach. In: Spilker, B., editor. Quality of life and pharmacoeconomics in clinical trials. Philadelphia: Lippincott-Raven; 1996. p. 41-8.

8. Juniper, EF.; Guyatt, GH.; Jaeschke, R. Chapter 6: How to develop and validate a new healthrelated quality of life instrument. In: Spilker, B., editor. Quality of life and pharmacoeconomics in clinical trials. Philadelphia: Lippincott-Raven; 1996. p. 49-56.

9. Cunningham SJ, Garratt AM, Hunt NP. Development of a condition-specific quality of life measure for patients with dentofacial deformity: II. Validity and responsiveness testing. Community Dent Oral Epidemiol. 2002; 30:81-90. [PubMed: 12000348]

10. Alexander M, Berger W, Buchholz P, Walt J, Burk C, Lee J, et al. The reliability, validity, and preliminary responsiveness of the Eye Allergy Patient Impact Questionnaire (EAPIQ). Health and Quality of Life Outcomes. 2005; 3:67. (Provisional PDF). [PubMed: 16259630]

11. Von Ranson KM, Klump KL, Iacono WG, McGue M. The Minnesota Eating Behavior Survey: a brief measure of disordered eating attitudes and behaviors. Eating Behav. 2005; 6:373-92.

12. Van Lang NDJ, Ferdinand RF, Oldehinkel AJ, Ormel J, Verhulst FC. Concurrent validity of the DSM-IV scales Affective Problems and Anxiety Problems of the Youth Self-Report. Behav Res Ther. 2005; 43:1485-94. [PubMed: 16159590]

13. Coyne K, Joshua-Gotlib S, Kimel M, Thompson C, Lewis A, Danilewitz M. Validation of the treatment satisfaction questionnaire for Crohn's Disease (TSQC0). Digestive Dis Sci. 2005; 50:252-8.

14. Aalto A, Harkapaa K, Aro AR, Rissanen P. Ways of coping with asthma in everyday life: validation of the Asthma Specific Coping Scale. J Psychosommatic Res. 2002; 53:1061-9.

15. Eisner MD, Trupin L, Katz PP, Yelin EH, Earnest G, Balmes J, et al. Development and validation of a survey-based COPD severity score. Chest. 2005; 127:1890-7. [PubMed: 15947299]

16. Richmond, S.; O’Brien, K.; Buchanan, I.; Burden, D. An introduction to occlusal indices. West Yorkshire, England: ORTHO-CARE (UK) Limited; 1994.

17. Phillips, C.; Bennett, ME. Chapter 25: Psychological ramifications of orthognathic surgery. In: Fonseca, RJ., editor. Oral and maxillofacial surgery. Philadelphia: W.B. Saunders Co; 2000. p. 506-34.

18. Bracken, BA. Mutidimensional self concept scale. Austin, TX: Pro-Ed; 1992.

19. La Greca, AM. Social anxiety scales for children and adolescents: manual and instructions for the SASC, SASC-R, SAS-A (adolescents) and parent versions of the scales. Miami, FL: University of Miami; 1998.

20. Cohen, J. Statistical power analysis for the behavioral sciences. New Jersey: Lawrence Erlbaum Associates; 1988. p. 78-80.

21. Sallis JF, Grossman RM, Pinski RB, Patterson T, Nader PR. The development of scales to measure social support for diet and exercise behaviors. Prevent Med. 1987; 16:825-36.

22. Van den Akker-Scheek I, Stevens M, Spriensma A, van Horn JR. Groningen Orthopaedic Social support scale: validity and reliability. J Adv Nurs. 2004; 47:57-63. [PubMed: 15186468]

23. Cunningham SJ, Garratt AM, Hunt NP. Development of a condition-specific quality of life measure for patients with dentofacial deformity: I. Reliability of the instrument. Community Dent Oral Epidemiol. 2000; 28:195-201. [PubMed: 10830646]

24. Ware JE, Sherbourne CD. The MOS 36-item short-form health survey (SF-36) I. Conceptual framework and item selection. Med Care. 1992; 30:473-83. [PubMed: 1593914]

25. Slade GD, Spencer AJ. Development and evaluation of the Oral Health Impact Profile. Community Dent Health. 1994; 11:3-11. [PubMed: 8193981]

26. Atchison K, Dolan T. Development of the geriatric oral health Assessment Index. J Dent Ed. 1990; 54:680-87. 
27. Trulsson U, Strandmark M, Mohlin S, Berggren U. A qualitative study of teenagers' decisions to undergo orthodontic treatment with fixed appliance. J Orthodontics. 2002; 29:197-204.

28. Pope AW, Ward J. Factors associated with peer social competence in preadolescents with craniofacial anomalies. J Pediatr Psychol. 1997; 22:455-69. [PubMed: 9302844] 


\section{Table 2}

Descriptive statistics for the COHIP and standardized, domain-specific questionnaires (Dento-facial image, self-concept, and social anxiety)

\begin{tabular}{|c|c|c|c|c|}
\hline & \multicolumn{2}{|c|}{ Study sample } & \multicolumn{2}{|c|}{ Normative values } \\
\hline & Mean & SD & Mean & SD \\
\hline \multicolumn{5}{|l|}{ COHIP domains 1} \\
\hline Oral health & 26.0 & 5.6 & 25.4 & 6.5 \\
\hline Functional well-being & 20.3 & 3.4 & 19.5 & 4.3 \\
\hline Social-emotional well-being & 25.6 & 5.7 & 24.5 & 7.4 \\
\hline School/environment & 12.9 & 2.7 & 14.0 & 2.7 \\
\hline Self-image & 15.0 & 3.3 & 16.1 & 5.1 \\
\hline Treatment expectations & 5.8 & 1.8 & 5.1 & 1.8 \\
\hline \multicolumn{5}{|l|}{ Domain-specific questionnaires } \\
\hline Dento-facial image & 3.4 & 0.9 & & \\
\hline \multicolumn{5}{|l|}{ Self-concept ${ }^{2}$} \\
\hline Physical & 102.1 & 16.9 & 100 & 15 \\
\hline Social & 105.1 & 16.2 & 100 & 15 \\
\hline Affect & 106.9 & 16.3 & 100 & 15 \\
\hline Competence & 105.1 & 14.5 & 100 & 15 \\
\hline Academic & 105.9 & 16.3 & 100 & 15 \\
\hline Family & 104.6 & 15.0 & 100 & 15 \\
\hline \multicolumn{5}{|l|}{ Social anxiety ${ }^{3}$} \\
\hline FNE & 17.1 & 6.8 & 19.7 & 7.0 \\
\hline SADS-new & 13.7 & 4.7 & 13.2 & 4.2 \\
\hline SADS-general & 6.7 & 2.6 & 10.2 & 3.8 \\
\hline
\end{tabular}

1 Normative mean and SD taken from Broder HL, Wilson-Genderson M, Janal M. Reliability and convergent and discriminant validity of the Child Oral Health Impact Profile (COHIP Child's Version). Community Dent Oral Epidemiol.

2 A standard score between 86-115 indicates average self concept. Standardization procedures described in Bracken BA. Mutidimensional Self Concept Scale. Austin, TX: Pro-Ed, 1992.

3 Normative mean and SD taken from Table 6, Sample F in LaGreca AM, Social Anxiety Scales for Children@ $\odot$ and Adolescents@ 9 . Manual and Instructions for the SASC, SASC-R, SAS-A, and Parent versions of the scales. University of Miami, 1998. 
